\title{
Scattering of Spatial Optical Solitons by Subwavelength Air Holes
}

\author{
Jethro H. Greene and Allen Taflove, Fellow, IEEE
}

\begin{abstract}
Using the finite-difference time-domain method, we model the propagation of spatial optical solitons having two orthogonal electric field vector components, and the scattering of such solitons by compact subwavelength air holes (i.e., abrupt dielectric discontinuities in the direct paths of the solitons). Our propagation and scattering studies assume a realistic glass characterized by a three-pole Sellmeier linear dispersion, an instantaneous Kerr nonlinearity, and a dispersive Raman nonlinearity. An unexpected spatial soliton scattering phenomenon is observed: the coalescence of the scattered electromagnetic field into a propagating lower-energy spatial soliton at a point many tens of wavelengths beyond the scattering air hole. Overall, our computational technique is general, and should permit future investigations and design of devices exploiting spatial soliton interactions in background media having submicrometer air holes and dielectric and metal inclusions.
\end{abstract}

Index Terms-Finite-difference time-domain (FDTD), nonlinear Schrödinger (NLS), unidirectional pulse propagation equation (UPPE).

\section{INTRODUCTION}

$\mathbf{T}$ HE integrable cubic nonlinear Schrödinger (NLS) equation is a proper model for temporal soliton propagation in optical fibers, but can be inappropriate for spatial solitons, in which much higher power is required [1]. Integrable models approximate physical systems, but it is often necessary to account for the effects produced by the nonintegrability of the underlying nonlinear equations [1]. The generalized NLS equation extends to more than cubic media, but cannot be solved using the inverse scattering method, because the model is not integrable [2]-[4]. Analysis of spatial solitons in non-Kerr media requires a model more general than the cubic NLS equation [5], which requires the paraxial approximation and neglects both the vector nature of the electromagnetic field and polarization effects [2].

Recently, Kolesik et al. developed a unidirectional pulse propagation equation (UPPE) model, which provides a transition between envelope-based models and Maxwell's equations [6]. UPPE models can incorporate both linear and nonlinear polarizations, and have been used to model pulse propagation with arbitrary polarizations over distances of tens of meters. However, the UPPE model requires the absence of sharp optical interfaces intersecting the direction of propagation [6], and is therefore unable to model the interactions of beams with subwavelength material inhomogeneities. Also, Ciattoni et

Manuscript received May 1, 2007; revised July 14, 2007.

The authors are with the Department of Electrical Engineering and Computer Science, Northwestern University, Evanston, IL 60208-3118 USA (e-mail: jhgreen@ece.northwestern.edu).

Color versions of one or more of the figures in this letter are available online at http://ieeexplore.ieee.org.

Digital Object Identifier 10.1109/LMWC.2007.908041 al. derived a perfect optical soliton (POS) solution for spatial soliton propagation in Kerr nonlinear materials from Maxwell's equations [7]. The POS solution does not require the paraxial approximation, but cannot describe nonintegrable models.

The finite-difference time-domain (FDTD) method [8] potentially models all of the vector components of the electromagnetic field at the space-time scale of the carrier oscillation, while including all relevant higher-order effects [2]. Furthermore, there are no restrictions on sharp interfaces and the interaction of beams with arbitrary geometries can be modeled. We recently reported the general vector auxiliary differential equation (GVADE) formulation of FDTD [9], a general method which we believe to be the first technique to model electromagnetic wave propagation with two or three orthogonal electric field vector components in dispersive nonlinear materials, that can be applied to any geometry.

In this letter, we validate the GVADE FDTD method with two test-cases: a wide NLS equation solution that propagates as a fundamental soliton, and the propagation of a narrow NLS equation solution which periodically focuses and defocuses. We then apply the GVADE FDTD method to study the propagation of spatial optical solitons having two orthogonal electric field vector components, and the scattering of such solitons by compact subwavelength air holes. Our propagation and scattering studies assume a realistic glass background material characterized by a three-pole Sellmeier linear dispersion, an instantaneous Kerr nonlinearity, and a dispersive Raman nonlinearity. An unexpected soliton scattering phenomenon is observed: the coalescence of the scattered electromagnetic field into a propagating lower-energy spatial soliton at a point many tens of wavelengths beyond the air hole.

\section{General Vector AuXiLiary DifFerential EQUATION FDTD METHOD}

This section briefly summarizes recent progress in auxiliary differential equation (ADE) techniques, which extend the FDTD method to incorporate linear and nonlinear polarization by time-stepping auxiliary differential equations synchronously with Maxwell's curl equations [8]. Reformulated ADE FDTD methods have been reported which eliminate the need to solve a system of $N$ equations at each time-step, where $N$ is the number of poles of the chromatic dispersion [10], [11]. In [9], we reported in detail the GVADE FDTD method, which extends this technique to nonlinear optics problems where the electric field has two or three orthogonal vector components and validated the GVADE FDTD method for the case of temporal soliton propagation. This was an advance over previous FDTD Maxwell's equations models of optical solitons [12], which included only the nonlinear Kerr polarization, and described only a single electric field vector component. 


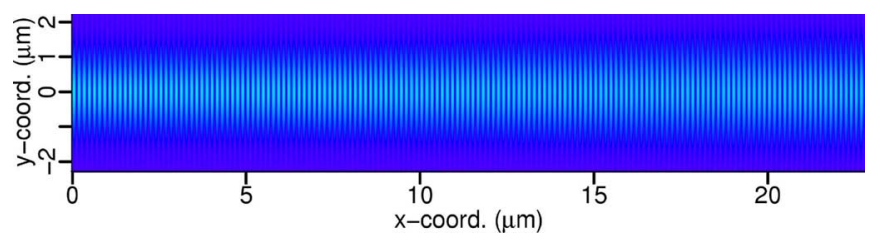

Fig. 1. Simulation results showing $|\mathbf{E}|$ for a wide spatial soliton with field components $\left\{E_{x}, E_{y}, H_{z}\right\}$ in a glass characterized by a three-pole Sellmeier linear dispersion, an instantaneous Kerr nonlinearity, and a dispersive Raman nonlinearity. The beam is excited with a transverse profile of a fundamental soliton as predicted by the NLS equation with a FWHM beamwidth of $3.99 \lambda_{d}$.

\section{VALIDATION OF GVADE FDTD MODELING OF SPATIAL OPTICAL SOLITON PROPAGATION}

We first report two new validations of the GVADE FDTD Maxwell's equations solution technique to model spatial optical soliton propagation in a realistic glass characterized by a three-pole Sellmeier linear dispersion, an instantaneous Kerr nonlinearity, and a dispersive Raman nonlinearity.

We assume the $+x$-directed propagation of a beam with the initial transverse profile of an NLS fundamental soliton with the electromagnetic field vector components $\left\{E_{x}, E_{y}, H_{z}\right\}$ having a carrier frequency of $\omega_{c}=4.35 \times 10^{15} \mathrm{rad} / \mathrm{s}\left(\lambda_{0}=433 \mathrm{~nm}\right)$. We assume a three-pole set of linear Sellmeier dispersions and the instantaneous Kerr and dispersive Raman nonlinearities used by Nakamura et al. [13]. The strengths and resonant frequencies of the linear dispersions from Sellmeier's equation are given by: $\beta_{1}=0.69617, \beta_{2}=0.40794, \beta_{3}=0.89748, \omega_{1}=2.7537 \times$ $10^{16} \mathrm{rad} / \mathrm{s}, \omega_{2}=1.62047 \times 10^{16} \mathrm{rad} / \mathrm{s}$, and $\omega_{3}=1.90342 \times$ $10^{14} \mathrm{rad} / \mathrm{s}$, which correspond to a linear index of refraction at the carrier frequency, $\omega_{c}$, of $n_{0}=1.47$. From Nakamura et al. [13], the nonlinear index coefficient is $n_{2}^{I}=2.48 \times$ $10^{-20} \mathrm{~m}^{2} / \mathrm{W}$, which, at the carrier frequency $\omega_{c}$, corresponds to a nonlinear index of refraction of $n_{2}=4.83 \times 10^{-23} \mathrm{~m}^{2} / \mathrm{V}^{2}$ and a third-order scalar electric susceptibility of $\chi_{0}^{(3)}=1.89 \times$ $10^{-22} \mathrm{~m}^{2} / \mathrm{V}^{2}$. The relative strengths of the Kerr and Raman polarizations are given by the parameter $\alpha=0.7$. The Raman polarization parameters are $\tau_{1}=12.2 \mathrm{fs}$ and $\tau_{2}=32 \mathrm{fs}$. We implement the model on a modified Yee grid having collocated electric field components [8]. For each simulation, the spatial and temporal resolutions were determined by numerical convergence tests.

\section{A. Validation of Fundamental Soliton Propagation: \\ Beamwidth Significantly Greater Than the Carrier Wavelength}

The first test case models a wide spatial soliton with a transverse profile (as provided by a solution of the NLS equation) having a full-width at half-maximum (FWHM) beamwidth of 3.99 dielectric wavelengths $\left(\lambda_{d}\right)$. This soliton is excited by a hard source at $x=0$ having a transverse profile $H_{z}(y, t)=$ $H_{0} \sin \left(\omega_{c} t\right) \operatorname{sech}(y / w)$, where $H_{0}=4.77 \times 10^{7} \mathrm{~A} / \mathrm{m}, \omega_{c}=$ $4.35 \times 10^{15} \mathrm{rad} / \mathrm{s}\left(\lambda_{0}=433 \mathrm{~nm}\right)$, and $w=667 \mathrm{~nm}$. The beam is sufficiently wide relative to $\lambda_{d}$ to propagate as a fundamental soliton, as predicted by the NLS equation. The grid for this simulation is 3600 by 2092 cells, with a spatial resolution of $\Delta x=\Delta y=\Delta=10 \mathrm{~nm}$ and a temporal resolution of $\Delta t=4.17 \times 10^{-18}$ s. The pseudocolor visualization of Fig. 1 shows that the soliton propagates without change to its transverse profile, as predicted by the NLS equation [2]. (Note that all visualizations utilize a common color scale.)
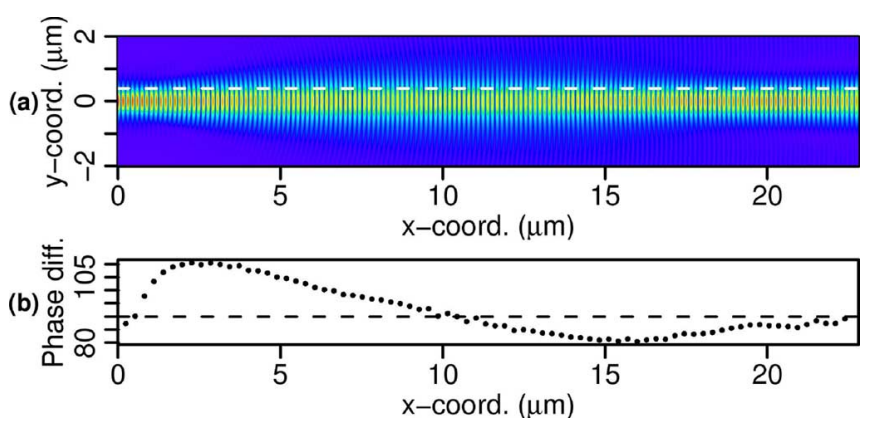

Fig. 2. Simulation results of soliton propagation as in Fig. 1, but for a narrow soliton having a FWHM beamwidth of $1.28 \lambda_{d}$ : (a) visualization of the spatial distribution of $|\mathbf{E}|$ and (b) phase difference between $E_{x}$ and $E_{y}$ along the white dashed line of (a) located at $y=395 \mathrm{~nm}$ from the center.

\section{B. Validation of Periodic Focusing and Defocusing: Beamwidth Approximately Equal to the Carrier Wavelength}

The second test case models a narrow spatial soliton with a transverse profile (as provided by a solution of the NLS equation) having a FWHM beamwidth of $1.28 \lambda_{d}$. This soliton is excited by a hard source having an initial transverse profile $H_{z}(y, t)=H_{0} \sin \left(\omega_{c} t\right) \operatorname{sech}(y / w)$, where $H_{0}=1.49 \times 10^{8} \mathrm{~A} / \mathrm{m}, \omega_{c}=4.35 \times 10^{15} \mathrm{rad} / \mathrm{s}\left(\lambda_{0}=\right.$ $433 \mathrm{~nm}$ ), and $w=215 \mathrm{~nm}$. Fig. 2 illustrates the GVADE FDTD computed propagation of the spatial optical soliton model summarized above. The grid for the simulation displayed in Fig. 2 is 5544 by 3451 cells with a spatial resolution of $\Delta x=\Delta y=\Delta=5.71 \mathrm{~nm}$ and a temporal resolution of $\Delta t=2.38 \times 10^{-18} \mathrm{~s}$. From Fig. 2(a), we see that the computed solution exhibits a periodic defocusing and focusing, despite its nominal fundamental-mode excitation. The narrow-soliton results demonstrate behavior predicted in [3] and [4], and show a defocusing perturbation that causes expansion followed by focusing leading to periodic oscillations. Fig. 2(b) shows that the phase difference between the longitudinal $E_{x}$ and transverse $E_{y}$ field components varies significantly around $90^{\circ}$ as the beam expands and contracts. Fig. 2 illustrates the GVADE FDTD computed propagation of the spatial optical soliton model summarized above.

\section{GVADE FDTD SimUlation OF WIDE, OVER-POWERED SPATIAL SOLITON PROPAGATION}

Having presented two validations of GVADE FDTD modeling of spatial soliton propagation, we next explore the propagation of wide, over-powered solitons with hyperbolic secant transverse profiles, as determined by solution of the NLS equation, but with twice the amplitude. (Electromagnetic field components $\left\{E_{x}, E_{y}, H_{z}\right\}$ and material properties are identical to those described in Section III; grid $\Delta=8 \mathrm{~nm}$ and $\Delta t=3.34 \times$ $10^{-18} \mathrm{~s}$.) Although the beamwidths are large relative to the carrier wavelength, the beams are found to propagate with a periodic focusing and defocusing. Fig. 3 shows that the period of the expansion-contraction cycle of the propagating spatial soliton diminishes as the beamwidth is reduced.

\section{GVADE FDTD SimUlation OF WIDE, OVER-PowERED SOLITON COLLISIONS WITH AIR HoleS}

Finally, we investigate the scattering of spatial solitons by abrupt, subwavelength, material discontinuities. Specifically, we consider the collision of the wide, over-powered soliton 

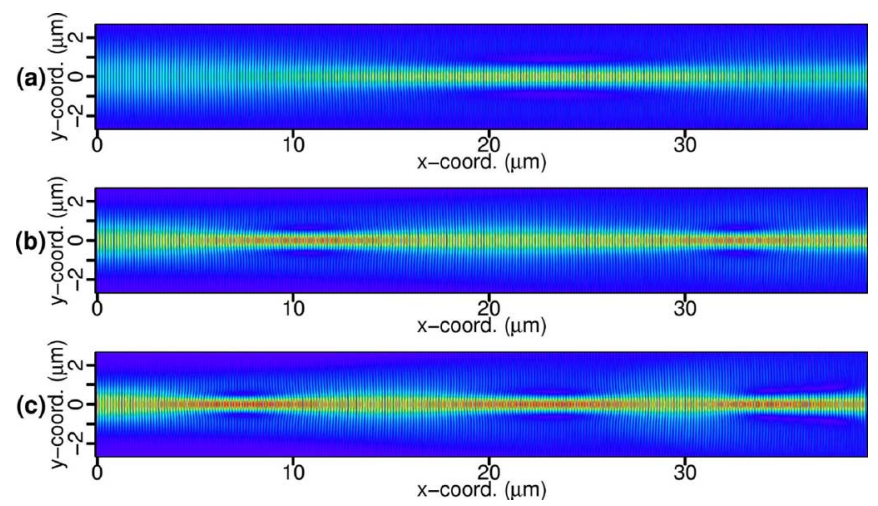

Fig. 3. Simulation of spatial soliton propagation as in Figs. 1 and 2 but with twice the amplitude indicated by the NLS equation. Visualization of $|\mathbf{E}|$ with three excitation beamwidths: (a) $5.98 \lambda_{d}$; (b) $3.99 \lambda_{d}$; and (c) $3.26 \lambda_{d}$.

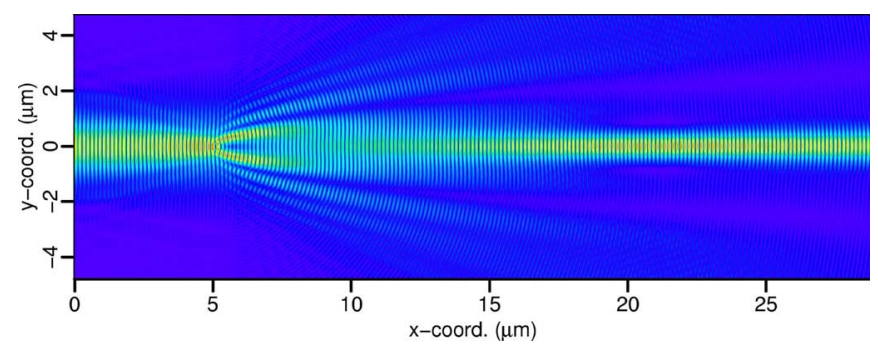

Fig. 4. Simulation of the collision of the over-powered soliton of Fig. 3(b) with a 250 by $250 \mathrm{~nm}$ air hole at $x=5 \mu \mathrm{m}$. Visualization of $|\mathbf{E}|$ showing the collision, diffraction, and subsequent reformation of a lower-power spatial soliton at a distance of approximately $30 \lambda_{d}$ beyond the air hole.

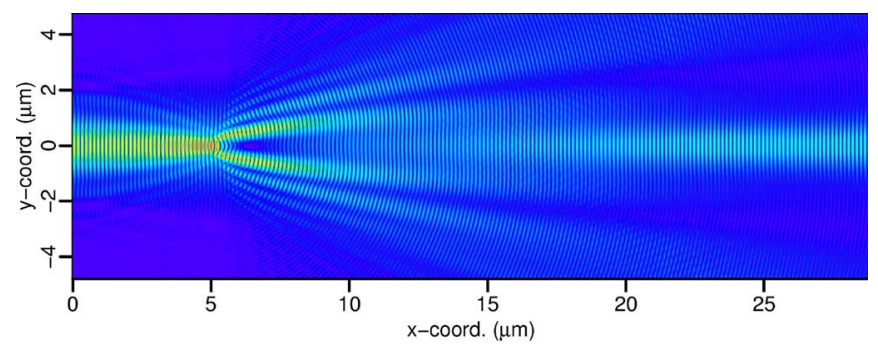

Fig. 5. Simulation of a soliton/air-hole collision as in Fig. 4, but with a larger air hole of size 350 by $350 \mathrm{~nm}$. The scattered electromagnetic field coalesces into a lower-power fundamental spatial soliton at a distance of approximately $60 \lambda_{d}$ beyond the scattering air hole.

shown in Fig. 3(b) with 250 and $350 \mathrm{~nm}$ square air holes at $x=$ $5 \mu \mathrm{m}$ in Fig. 3(b), at which point the soliton is at its average width. (Here, $\Delta=10 \mathrm{~nm}$ and $\Delta t=4.17 \times 10^{-18}$ s.) Figs. 4 and 5 illustrate significant scattering of the soliton electromagnetic field. We see that after each collision, the electromagnetic field energy that does not escape, coalesces into relatively lower-power solitons. In Fig. 4, a narrow soliton is reformed after a distance of approximately $30 \lambda_{d}$ beyond the scattering air hole. The reformed soliton exhibits periodic focusing and defocusing similar to Fig. 3. In Fig. 5, more energy is lost due to scattering by the larger air hole, and coalescence into a reformed soliton occurs at a distance of approximately $60 \lambda_{d}$ beyond the air hole. Here, the lower-power reformed soliton propagates as a fundamental soliton, i.e., without periodic focusing and defocusing.

\section{CONCLUSION}

In this letter, we applied the GVADE formulation of FDTD to study the propagation of spatial optical solitons having two orthogonal electric field vector components in a realistic glass, and the scattering of such solitons by compact subwavelength air holes. An unexpected spatial soliton scattering phenomenon was observed: the coalescence of the scattered electromagnetic field into a propagating lower-energy spatial soliton at a point many tens of wavelengths beyond the scattering air hole.

The technique applied here is general, and can model the multicomponent vector electric field of spatial solitons scattering from abrupt, arbitrary-shaped, subwavelength dielectric and metal discontinuities. This appears to be a novel capability permitting future investigations and design of devices exploiting spatial soliton interactions in background media having submicrometer air holes and dielectric and metal inclusions.

\section{REFERENCES}

[1] D. E. Pelinovsky, Y. S. Kivshar, and V. V. Afanasjev, "Internal modes of envelope solitons," Phys. D, vol. 116, no. 1-2, pp. 121-142, May 1998.

[2] Y. S. Kivshar and G. P. Agrawal, Optical Solitons: From Fibers to Photonic Crystals. San Diego, CA: Academic, 2003, ch. 2, 6, 7.

[3] A. W. Snyder, S. J. Hewlett, and D. J. Mitchell, "Periodic solitons in optics," Phys. Rev. E, vol. 51, no. 6, pp. 6297-6300, Jun. 1995.

[4] G. Fibich and G. Papanicolaou, "A modulation method for self-focusing in the perturbed critical nonlinear Schrödinger equation," Phys. Lett. A, vol. 239, no. 3, pp. 167-173, Mar. 1998.

[5] Y. S. Kivshar, D. E. Pelinovsky, T. Cretegny, and M. Peyrard, "Internal modes of solitary waves," Phys. Rev. Lett., vol. 80, no. 23, pp. 5032-5035, Jun. 1998.

[6] M. Kolesik and J. V. Moloney, "Nonlinear optical pulse propagation simulation: From Maxwell's to unidirectional equations," Phys. Rev. E, vol. 70, p. 036604, Sep. 2004.

[7] A. Ciattoni, B. Crosignani, P. D. Porto, and A. Yariv, "Perfect optical solitons: Spatial Kerr solitons as exact solutions of Maxwell's equations," J. Opt. Soc. Amer. B, vol. 22, no. 7, pp. 1384-1394, Jul. 2005.

[8] A. Taflove and S. C. Hagness, Computational Electrodynamics: The Finite-Difference Time-Domain Method, 3rd ed. Norwood, MA: Artech House, 2005, ch. 3, 7, 9.

[9] J. H. Greene and A. Taflove, "General vector auxiliary dif-ferential equation finite-difference time-domain method for nonlinear optics," Opt. Express, vol. 14, pp. 8305-8310, 2006.

[10] S. Nakamura, Y. Koyamada, N. Karasawa, H. Sone, R. Morita, and M. Yamashita, "Finite-difference time-domain analysis of ultrashort laser pulse propagation in a fiber with nonlinear effects," Electron. Comm. Jpn. 1, vol. 87, no. 5, pp. 1-10, May 2004.

[11] M. Fujii, M. Tahara, I. Sakagami, W. Freude, and P. Russer, "Highorder FDTD and auxiliary differential equation formulation of optical pulse propagation in 2-D Kerr and Raman nonlinear dispersive media," IEEE J. Quant. Electron., vol. 40, no. 2, pp. 175-182, Feb. 2004.

[12] R. M. Joseph, S. C. Hagness, and A. Taflove, "Direct time integration of Maxwell's equations in linear dispersive media with absorption for scattering and propagation of femtosecond electromagnetic pulses," Opt. Lett., vol. 16, no. 18, pp. 1412-1414, Sep. 1991.

[13] S. Nakamura, N. Takasawa, and Y. Koyamada, "Comparison between finite-difference time-domain calculation with all parameters of Sellmeier's fitting equation and experimental results for slightly chirped 12 fs laser pulse propagation in a silica fiber," IEEE J. Lightw. Technol., vol. 23, no. 2, pp. 855-863, Feb. 2005. 